\title{
Clinical Outcomes of Multiple Osteochondral Allograft Transplantation of the Knee: An Analysis of Snowman Technique and Multifocal Lesions
}

Eric J. Cotter, BS ${ }^{1}$, Charles P. Hannon, MD², Drew A. Lansdown, MD³, Rachel M. Frank, MD4, Brian Robert Waterman, $M^{5}$, Brian J. Cole, MD, MBA 6

${ }^{1}$ Georgetown University School of Medicine, Washington, DC, USA, 2Rush University Medical Center, Chicago, IL, USA, ${ }^{3}$ University of California-San Francisco, San Francisco, CA, USA, ${ }^{4}$ University of Colorado School of Medicine, Boulder, CO, USA, ${ }^{5}$ Wake Forest University School of Medicine, Winston Salem, NC, USA, ${ }^{6}$ Midwest Orthopaedics at Rush, Chicago, IL, USA.

Objectives: To report the clinical outcomes of snowman technique osteochondral allograft transplantation (OCA) and clinical outcomes of multifocal OCA.

Methods: Consecutive patients who underwent either a primary snowman OCA or multifocal (i.e. bipolar patellofemoral, patellofemoral and a condyle or bicondylar) with a minimum 2-year follow-up by a single surgeon from 4/2003 to 4/2015 were isolated. Failure was defined as revision OCA, conversion to arthroplasty, or gross appearance of graft degeneration on $2^{\text {nd }}$ look arthroscopy.

Results: Twenty-six patients (28 knees) were isolated with 22 patients (24 knees; $85.7 \%$ ) having 2-year clinical follow-up. Nine of 11 patients $(81.8 \%)$ who underwent isolated condylar snowman allografts met inclusion criteria at mean follow-up of $7.4 \pm 3.6$ years, while 13 additional patients (15 knees; $88.2 \%)$ underwent multifocal OCA at mean follow-up of $6.4 \pm 3.9$ years. All 9 patients who received isolated snowman OCA were to the medial femoral condyle. Reoperations were common with $44.4 \%(\mathrm{~N}=4)$ of the snowman group and $20 \%$ of multifocal OCA $(\mathrm{N}=3)$ undergoing at least 1 reoperation. There were 3 failures $(33.3 \%)$ in the snowman technique group at a mean $7.7 \pm 5.5$ years and 1 failure in the multifocal OCA group at 4.5 years. All 4 failures underwent TKA. Patients who underwent multifocal OCA demonstrated significant improvements in the International Knee Documentation Committee score, Knee Injury and Osteoarthritis subscores, Western Ontario and McMaster Universities Osteoarthritis Index subscores, and the Short-Form-12 physical component $(\mathrm{P}<0.05$ for all). Patients who underwent snowman OCA demonstrated significant improvement in KOOS pain subscore and WOMAC overall scores ( $P<0.05$ for both) Table 1]. Conclusion: While in a small cohort, patients who underwent snowman OCA had a high rate of reoperation $(44.4 \%)$ and a high rate of failure $(33.3 \%)$. Comparatively, patients who underwent multifocal OCA had reoperation and graft survival rates comparable to published literature for focal OCA.

This open-access article is published and distributed under the Creative Commons Attribution - NonCommercial - No Derivatives License (http://creativecommons.org/licenses/by-nc-nd/4.0/), which permits the noncommercial use, distribution, and reproduction of the article in any medium, provided the original author and source are credited. You may not alter, transform, or build upon this article without the permission of the Author(s). For article reuse guidelines, please visit SAGE's website at http://www.sagepub.com/journals-permissions. 
Table 1: Patient-reported clinical outcome measures for both snowman technique osteochondral allografts and multifocal grafts

Multifocal Osteochondral Allograft Transplantation

\begin{tabular}{|l|r|r|r|r|r|}
\hline & \multicolumn{2}{|c|}{ Preoperative } & \multicolumn{2}{|c|}{ Postoperative } & \multicolumn{1}{l|}{ P Value } \\
\hline & Mean & \multicolumn{1}{|c|}{ SD } & \multicolumn{1}{c|}{ Mean } & \multicolumn{1}{|c|}{ SD } & \\
\hline Marx & 1.67 & 1.60 & 6.13 & 3.57 & 0.285 \\
\hline Lysholm & 41.83 & 9.31 & 55.00 & 13.62 & 0.237 \\
\hline IKDC & 32.71 & 5.02 & 52.92 & 12.55 & $\mathbf{0 . 0 1 0}$ \\
\hline KOOS-Pain & 51.48 & 9.59 & 65.28 & 10.57 & 0.155 \\
\hline KOOS - Symptom & 56.25 & 9.99 & 66.07 & 9.47 & $\mathbf{0 . 0 2 5}$ \\
\hline KOOS - ADL & 58.70 & 9.47 & 77.21 & 10.18 & $\mathbf{0 . 0 2 6}$ \\
\hline KOOS - Sport & 18.33 & 9.19 & 40.36 & 14.66 & $\mathbf{0 . 0 2 6}$ \\
\hline KOOS - QOL & 11.98 & 5.41 & 41.07 & 16.16 & $\mathbf{0 . 0 0 5}$ \\
\hline WOMAC - Pain & 8.82 & 1.81 & 6.07 & 1.81 & 0.212 \\
\hline WOMAC - Stiffness & 4.00 & 0.89 & 2.93 & 0.89 & $\mathbf{0 . 0 4 6}$ \\
\hline WOMAC - Function & 28.18 & 5.20 & 15.36 & 6.79 & $\mathbf{0 . 0 3 7}$ \\
\hline WOMAC - Overall & 37.55 & 8.73 & 15.93 & 7.96 & $\mathbf{0 . 0 4 7}$ \\
\hline SF-12 Physical & 33.62 & 4.07 & 44.65 & 5.25 & $\mathbf{0 . 0 1 1}$ \\
\hline SF-12 Mental & 53.14 & 5.10 & 55.11 & 3.37 & 0.859 \\
\hline & & & & &
\end{tabular}

Snowman Technique Osteochondral Allograft Transplantation

\begin{tabular}{|l|r|r|r|r|r|}
\hline Marx & 0.00 & 0.00 & 2.33 & 0.76 & N/A \\
\hline Lysholm & 37.17 & 3.83 & 66.17 & 14.75 & 0.138 \\
\hline IKDC & 29.34 & 2.54 & 54.14 & 13.29 & 0.285 \\
\hline KOOS-Pain & 44.91 & 5.51 & 72.62 & 9.77 & 0.043 \\
\hline KOOS - Symptom & 46.43 & 5.30 & 66.33 & 11.24 & 0.080 \\
\hline KOOS - ADL & 56.86 & 13.25 & 82.35 & 11.09 & 0.345 \\
\hline KOOS - Sport & 17.50 & 3.06 & 53.57 & 19.35 & 0.223 \\
\hline KOOS - QOL & 19.79 & 7.24 & 49.11 & 14.71 & 0.104 \\
\hline WOMAC - Pain & 9.17 & 1.93 & 6.00 & 2.47 & 0.109 \\
\hline WOMAC - Stiffness & 4.83 & 0.86 & 2.71 & 0.75 & 0.068 \\
\hline WOMAC - Function & 29.50 & 9.08 & 12.00 & 7.43 & 0.345 \\
\hline WOMAC -Overall & 45.17 & 10.45 & 10.71 & 6.18 & 0.043 \\
\hline SF-12 Physical & 45.01 & 3.03 & 41.45 & 5.40 & 0.686 \\
\hline SF-12 Mental & 52.61 & 5.13 & 55.71 & 7.48 & 0.500 \\
\hline
\end{tabular}

The Orthopaedic Journal of Sports Medicine, 6(7)(suppl 4)

DOI: $10.1177 / 2325967118 S 00093$

(C)The Author(s) 2018 